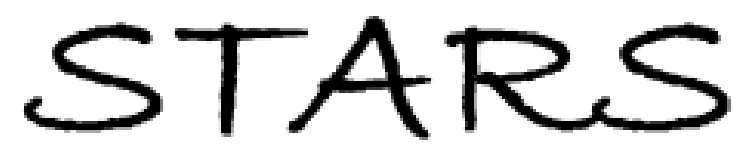

University of Central Florida

STARS

$1-1-1995$

\title{
Spectroscopic Characteristics Of Nd3+-Doped Strontium Fluorovanadate And Their Relationship To Laser Performance
}

\author{
P. Hong \\ University of Central Florida \\ X. X. Zhang \\ University of Central Florida \\ R. E. Peale \\ University of Central Florida \\ H. Weldner \\ University of Central Florida \\ M. Bass \\ University of Central Florida
}

Find similar works at: https://stars.library.ucf.edu/facultybib1990

University of Central Florida Libraries http://library.ucf.edu

See next page for additional authors

This Article is brought to you for free and open access by the Faculty Bibliography at STARS. It has been accepted for inclusion in Faculty Bibliography 1990s by an authorized administrator of STARS. For more information, please contactSTARS@ucf.edu.

\section{Recommended Citation}

Hong, P.; Zhang, X. X.; Peale, R. E.; Weldner, H.; Bass, M.; and Chai, H. T., "Spectroscopic Characteristics Of Nd3+-Doped Strontium Fluorovanadate And Their Relationship To Laser Performance" (1995). Faculty Bibliography 1990s. 3000.

https://stars.library.ucf.edu/facultybib1990/3000

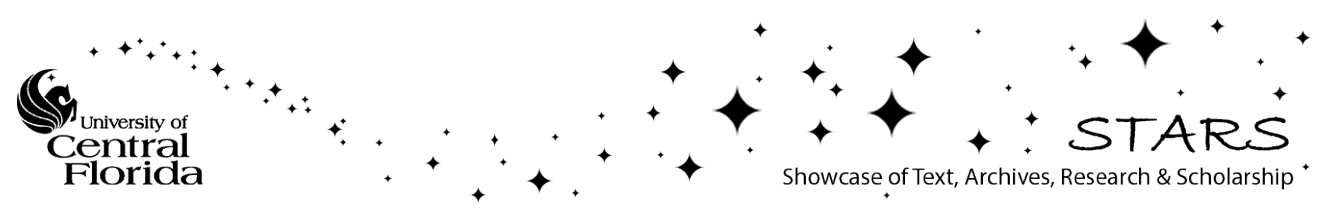


Authors

P. Hong, X. X. Zhang, R. E. Peale, H. Weldner, M. Bass, and H. T. Chai 


\section{Spectroscopic characteristics of $\mathrm{Nd}^{3+}$ - doped strontium fluorovanadate and their relationship to laser performance}

Cite as: Journal of Applied Physics 77, 294 (1995); https://doi.org/10.1063/1.359391

Submitted: 05 July 1994 . Accepted: 16 September 1994 . Published Online: 04 June 1998

P. Hong, X. X. Zhang, R. E. Peale, H. Weidner, M. Bass, and B. H. T. Chai

ARTICLES YOU MAY BE INTERESTED IN

Site-selective excitation and polarized absorption spectra of $\mathrm{Nd}^{3+}$ in $\mathrm{Sr}_{5}\left(\mathrm{PO}_{4}\right)_{3} \mathrm{~F}$ and $\mathrm{Ca}_{5}\left(\mathrm{PO}_{4}\right)_{3} \mathrm{~F}$

Journal of Applied Physics 79, 1746 (1996); https://doi.org/10.1063/1.360964

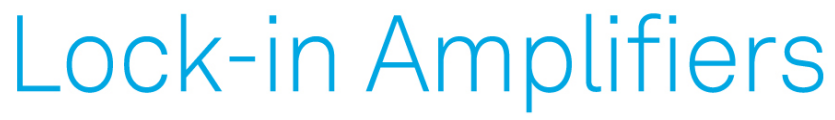

... and more, from DC to $600 \mathrm{MHz}$

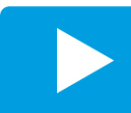

Watch 


\title{
Spectroscopic characteristics of $\mathrm{Nd}^{3+}$-doped strontium fluorovanadate and their relationship to laser performance
}

\author{
P. Hong, X. X. Zhang, R. E. Peale, H. Weidner, M. Bass, and B. H. T. Chai \\ CREOL-Center for Research and Education in Optics and Lasers, University of Central Florida, \\ 12424 Research Parkway, Orlando, Florida 32826
}

(Received 5 July 1994; accepted for publication 16 September 1994)

\begin{abstract}
High slope efficiency and low threshold laser performance have been achieved for both long pulsed and $\mathrm{cw}$ operation at $1.065 \mu \mathrm{m}$ in $\mathrm{Nd}^{3+}$-doped strontium fluorovanadate crystal, $\mathrm{Nd}^{3+}: \mathrm{Sr}_{5}\left(\mathrm{VO}_{4}\right)_{3} \mathrm{~F}$, when pumped by narrow band pulsed Cr:LiSAF and $\mathrm{cw}$ Ti:sapphire lasers. However, there are inequivalent $\mathrm{Nd}^{3+}$ sites in the crystal. The absorption of $\mathrm{Nd}^{3+}$ ions in secondary sites, sites other than the site which contributes to lasing, may reduce the pumping efficiency and, consequently, the lasing efficiency. Strong concentration quenching of the $\mathrm{Nd}^{3+}{ }^{4} \Gamma_{3 / 2}$ state was also obscrved reducing the quantum efficiency of the laser transition from this state. (C) 1995 American Institute of Physics.
\end{abstract}

\section{INTRODUCTION}

As efficient, high power diode lasers have become more readily available, there has been a renewed interest in the search for high efficiency and low threshold solid state laser media for diode laser pumping. The $\mathrm{Nd}^{3+}$-doped apatite crystals are attractive for this purpose because they are available as large, high quality crystals and have unique spectroscopic properties appropriate for diode pumping. ${ }^{1,2}$ This article presents the laser performance and related spectroscopic properties of a newly discovered laser crystal, ${ }^{3,4}$ neodymiumdoped strontium fluorovanadate, $\mathrm{Sr}_{5}\left(\mathrm{VO}_{4}\right)_{3} \mathrm{~F}$, or S-VAP.

$\mathrm{S}$-VAP is isomorphous to calcium fluoroapatite, $\mathrm{Ca}_{5}\left(\mathrm{PO}_{4}\right)_{3} \mathrm{~F}$ or $\mathrm{FAP},{ }^{5}$ which is hexagonal with a space group $P 6_{3} / m$ and unit cell dimensions of $a_{0}=10.0077 \AA$ and $c_{0}=7.4342 \AA$. Each unit cell contains two $\mathrm{Sr}_{5}\left(\mathrm{VO}_{4}\right)_{3} \mathrm{~F}$ molecules. Two crystallographically inequivalent $\mathrm{Sr}^{2+}$ sites, $M_{\mathrm{I}}\left(40 \%\right.$, nine-fold with $C_{3}$ symmetry) and $M_{\mathrm{II}}(60 \%$, sevenfold with $C_{1 h}$ symmetry), exist in each unit cell. Since the $M_{\text {II }}$ site has one $\mathrm{F}^{-}$in coordination, it is the dominant site for rare earth substitution. $\mathrm{An}^{\mathrm{Nd}^{3+}}$ ion substitutes for an $M_{\mathrm{II}}$ site $\mathrm{Sr}^{2+}$ ion in the structure with the unbalanced charge compensated by replacing $\mathrm{F}^{-}$ion with an $\mathrm{C}^{2-}$ ion. ${ }^{6} \mathrm{~A}$ small fraction of rare earth doping may also substitute in the $M_{\mathrm{I}}$ site as will be shown by our spectroscopic data. High quality single crystals were grown by conventional Czochralski pulling in our crystal growth laboratory. The growing conditions are similar to those used to grow its isomorph, FAP. ${ }^{2,7}$

Laser characteristics (i.e., gain, threshold, and slope efficiency) of a material are determined by its spectroscopic properties. Using absorption, emission, excitation, and site selective spectroscopy techniques, we have identified more than two inequivalent $\mathrm{Nd}^{3+}$ sites in Nd:S-VAP. The energy levels of the main emitting site were determined by high resolution spectroscopic analysis. The effects of the inequivalent sites on laser performance are analyzed and the concentration dependence of the decay time of $\mathrm{Nd}^{3+}$ ions in S-VAP is reported.

\section{SPECTROSCOPIC PROPERTIES}

Spectroscopic properties of $\mathrm{Nd}^{3+}: \mathrm{S}-\mathrm{VAP}$ were studied in several samples. The $\mathrm{Nd}^{3+}$ doping concentration in the melts were $0.2,1,1.5,2,3$, and 4 at. $\%$, respectively, but the respective $\mathrm{Nd}^{3+}$ concentrations in the grown crystals were found to be $0.05,0.26,0.38,0.48,0.63$, and 0.71 at. \% using $\mathrm{x}$-ray microprobe analysis. The distribution coefficient of $\mathrm{Nd}^{3+}$ ions in S-VAP crystal is different for different doping levels, decreasing as the $\mathrm{Nd}^{3+}$ doping level increases. That is, it is more difficult to dope $\mathrm{Nd}^{3+}$ into S-VAP crystal as the $\mathrm{Nd}^{3+}$ concentration increases.

The absorption and emission spectra were measured with a Fourier transform spectrometer. An argon ion laser and an argon ion laser pumped Ti:sapphire tunable laser were used as pumping sources in the steady state measurements. A tunable, $Q$-switched Cr:LiSrAlF 6 (Cr:LiSAF) laser was used in the luminescence dynamics measurements. The steady state luminescence measurements were made with a Si detector and the dynamic measurements employed an $S_{1}$ photomultiplier tube with nanosecond response time.

For a single site crystal two absorption lines for the ${ }^{4} I_{9 / 2} \rightarrow{ }^{4} F_{3 / 2}$ transition are expected at low temperature because of the crystal field splitting of the ${ }^{4} F_{3 / 2}$ state of $\mathrm{Nd}^{3+}$ ions in crystals. Polarized transmission spectra of the ${ }^{4} I_{9 / 2} \rightarrow{ }^{4} F_{3 / 2}$ transition at $2 \mathrm{~K}$ are shown in Fig. 1 with a resolution of $1 \mathrm{~cm}^{-1} . \mathrm{Nd}^{3+}$ ions seem to be in more than two inequivalent sites in S-VAP since at least 20 absorption. lines appear for this transition. The low-energy absorption lines in the spectra are sharp while the high-energy lines appear to be broader. As the $\mathrm{Nd}^{3+}$ concentration increases the relative absorption coefficient of the lines in the middle of the spectrum seems to decrease as indicated in Fig. 1. The emission spectra of the ${ }^{4} F_{3 / 2} \rightarrow{ }^{4} I_{11 / 2}$ transition at $20 \mathrm{~K}$ when pumped by a multiline argon ion laser are shown in Fig. 2 . The spectrum consists of one predominant emission line at $1.065 \mu \mathrm{m}$ and some weaker emission lines. At this temperature, only the lower level of the ${ }^{4} F_{3 / 2}$ manifold is populated. As a result, only six emission lines for the ${ }^{4} F_{3 / 2} \rightarrow{ }^{4} I_{11 / 2}$ transition are expected for a single site crystal. The fact that more than six emission lines are observed in Fig. 2 indicates once again the existence of more than one inequivalent site in S-VAP.

By studying the low temperature emission and absorption spectra involving transitions between the ${ }^{4} F_{3 / 2}$ and ${ }^{4} I_{9 / 2}$, ground state manifolds, one can identify the split sublevels 


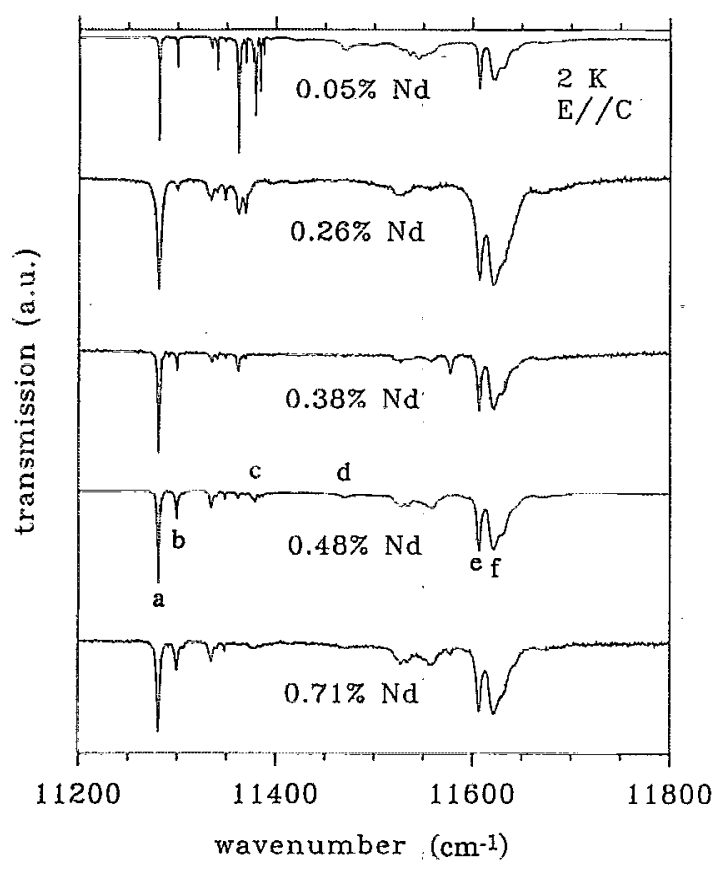

FIG. 1. $\pi$ polarized transmission spectra $\left({ }^{4} I_{9 / 2} \rightarrow{ }^{4} F_{3 / 2}\right)$ of $\mathrm{Nd}^{3+}: \mathrm{S}-\mathrm{VAP}$ at 2 $\mathrm{K}$ for different concentrations.

of the ${ }^{4} F_{3 / 2}$ manifold. In doing so we were able to identify those sharp lines in the lower energy side of the absorption spectra in Fig. 1 as resulting from transition to the lower sublevels of the ${ }^{4} F_{3 / 2}$ manifold of $\mathrm{Nd}^{3+}$ ions in different sites and those broader lines in the higher energy side of the spectra from transition to the higher sublevels. Eleven different sites were identified in this manner and their lower sublevel positions are listed in Table $\mathrm{I}$.

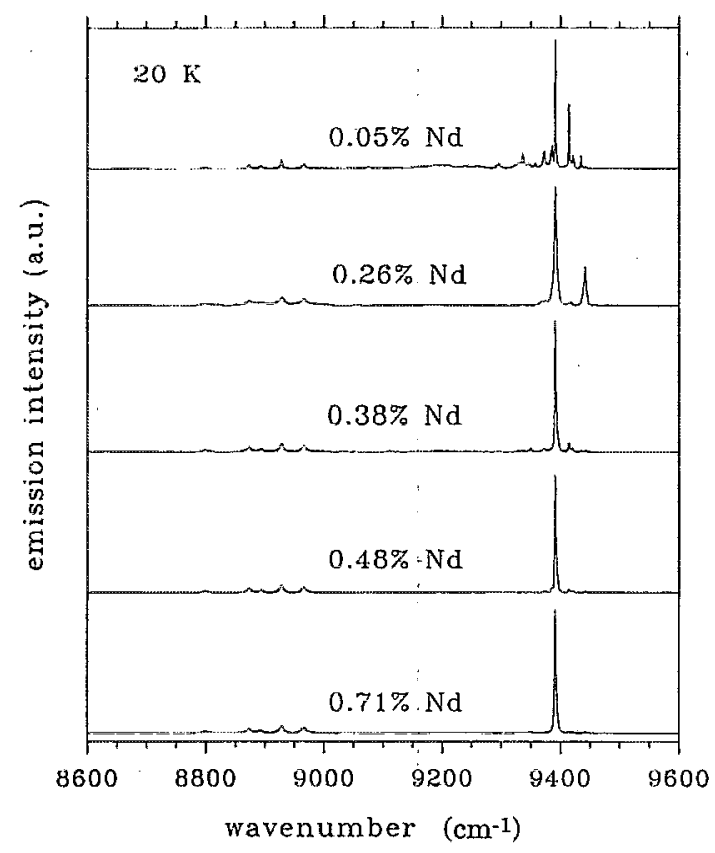

FIG. 2. Emission spectra $\left({ }^{4} F_{3 / 2} \rightarrow{ }^{4} I_{11 / 2}\right)$ of $\mathrm{Nd}^{3+}: \mathrm{S}-\mathrm{VAP}$ at $20 \mathrm{~K}$ for different concentrations excited by a multiline argon ion laser.
TABLE I. Energy positions of the 11 strongest inequivalent absorption lines from the lower ${ }^{4} F_{3 / 2}$ state of $\mathrm{Nd}^{3+}$ ion in S-VAP at $2 \mathrm{~K}$.

\begin{tabular}{lc}
\hline Spectral term & Wave number $\left(\mathrm{cm}^{-1}\right)$ \\
\hline Lower ${ }^{4} F_{3 / 2}$ & $11281,11300,11335,11340,11349,11362 ;$ \\
& $11369,11377,11379,11384,11387$ \\
\hline \hline
\end{tabular}

In order to understand the absorption and emission of these inequivalent sites, we conducted a site selective spectroscopy investigation with a $0.48 \% \mathrm{Nd}^{3+}$-doped S-VAP sample using a tunable Ti:sapphire laser. We selectively excited into the different ${ }^{4} I_{9 / 2} \rightarrow{ }^{4} F_{3 / 2}$ absorption lines which correspond to $\mathrm{Nd}^{3+}$ ions at different sites, and detected the emission spectrum of the ${ }^{4} F_{3 / 2} \rightarrow{ }^{4} I_{11 / 2}$ transition. We also measured the excitation spectra of the ${ }^{4} F_{3 / 2} \rightarrow{ }^{4} I_{11 / 2}$ emission at $1.065 \mu \mathrm{m}$.

As shown in Fig. 3(a), when the sample was excited at the peak of a line a $\left(11281 \mathrm{~cm}^{-1}\right)$ in Fig. 1, only six emission lines from the ${ }^{4} F_{3 / 2} \rightarrow{ }^{4} I_{11 / 2}$ transition were observed with the strongest line centered at $1.065 \mu \mathrm{m}$. For excitation at

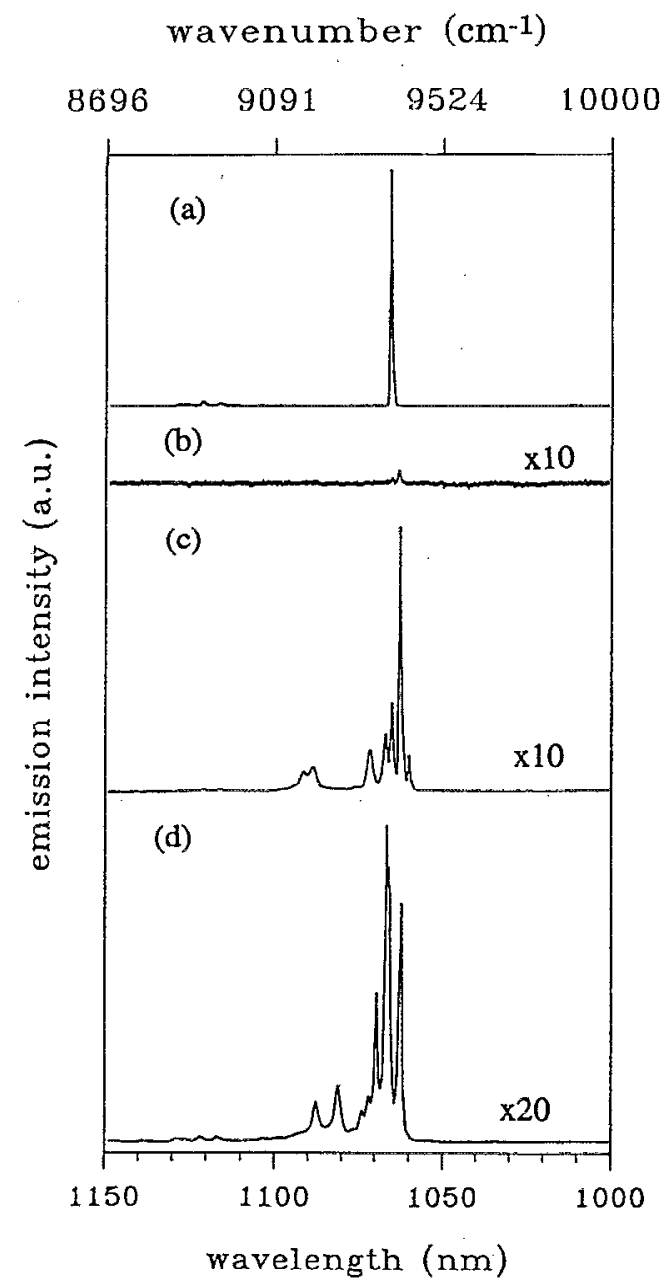

FIG. 3. Emission spectra $\left({ }^{4} F_{3 / 2} \rightarrow{ }^{4} I_{11 / 2}\right)$ of 0.48 at. $\% \mathrm{Nd}^{3+}: \mathrm{S}-\mathrm{VAP}$ at $15 \mathrm{~K}$ when excite at (a) $11281 \mathrm{~cm}^{-1}$, (b) $11300 \mathrm{~cm}^{-1}$, (c) $11366 \mathrm{~cm}{ }^{1}$, and (d) $11473 \mathrm{~cm}^{-1}$ 


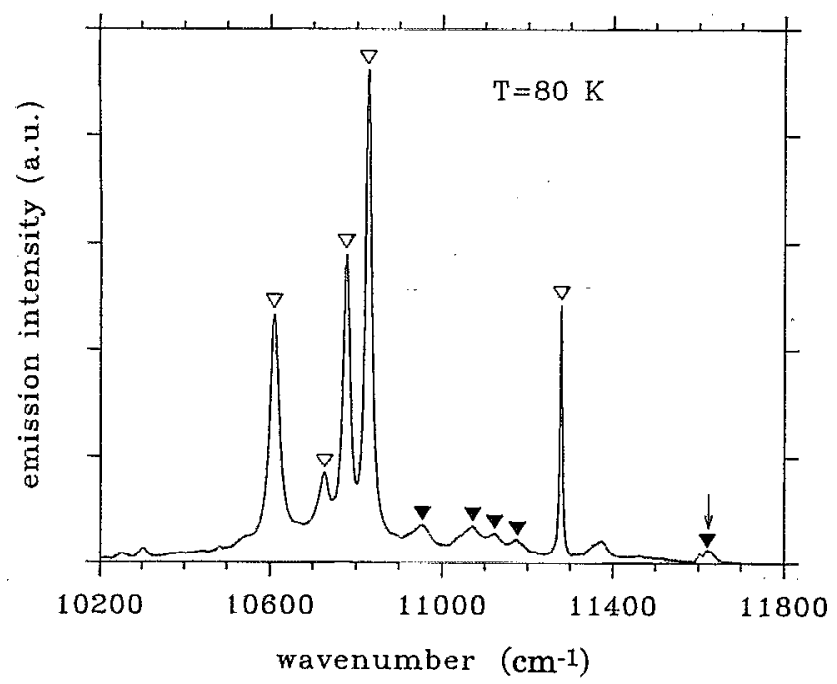

FIG. 4. $80 \mathrm{~K}$ emission spectra $\left({ }^{4} F_{3 / 2} \rightarrow{ }^{4} I_{9 / 2}\right)$ of 0.48 at. $\% \mathrm{Nd}^{3+}: \mathrm{S}$-VAP under the excitation of a multiline argon ion laser. The hollow and filled triangles indicate the emission lines from the lower and higher sublevel of ${ }^{4} F_{3 / 2}$, respectively. The arrow indicates the emission from lines $e$ and $f$ of Fig. 1.

lines $e$ and $f$, the same spectra were obtained as when we excited line $a$, indicating that lines $e$ and $f$ are either of the same origin or strongly coupled. However, the energy separation between line $a$ and line $f$ matches the separation between the $1.065 \mu \mathrm{m}$ emission line and its thermal replica at higher temperatures as we will see later. We therefore assign lines $a$ and $f$ to the split sublevels of the ${ }^{4} F_{3 / 2}$ manifold in a particular site. We further attribute this site to the main site, $M_{\text {II }}$ site, since its absorption and emission are the strongest observed.

Excitation at line $b\left(11300 \mathrm{~cm}^{-1}\right)$ in Fig. 1 resulted in a weak emission spectrum with the strongesst line centered at $1.063 \mu \mathrm{m}$, as seen in Fig. 3(b). For excitation at absorption lines between line $b$ and $e$, many weak emission lines, including the main emission line, were detected. The relative intensity of these weak emission lines depends on the excitation wavelength as seen in Fig. 3 curves (c) and (d). These absorption features are most probably due to ions in the $M_{\mathrm{I}}$ site and others that result from charge compensation, all of which we call secondary sites. The presence of the main emission line at $1.065 \mu \mathrm{m}$ under such excitation conditions may indicate that energy transfer takes place from the secondary sites tô the mâin site.

Absorption and emission spectra at 80 and $300 \mathrm{~K}$ were also measured. The absorption spectra at $80 \mathrm{~K}$ are almost identical to those at $2 \mathrm{~K}$. Emission spectrum of the ${ }^{4} F_{3 / 2} \rightarrow{ }^{4} I_{9 / 2}$ transition at $80 \mathrm{~K}$, as shown in Fig. 4, consists of the main emission lines (indicated by hollow triangles) and their thermal replicas (indicated by filled triangles). The energy difference between the emission lines from the lower ${ }^{4} F_{3 / 2}$ state and their respective thermal replicas from the upper ${ }^{4} F_{3 / 2}$ state matches the energy difference between absorption lines $a$ and $f$. shown in Fig. 1. Even though the nominal temperature is $80 \mathrm{~K}$ the actual temperature may be higher because the power of the argon ion laser used as ex-

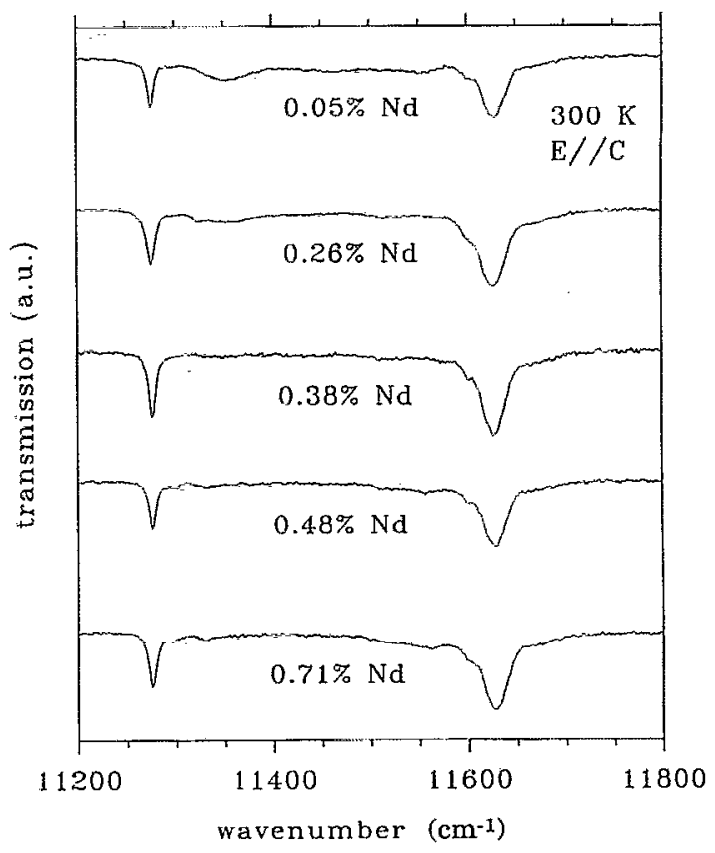

FIG. 5. Room temperature $\pi$ polarized transmission spectra $\left({ }^{4} I_{9 / 2} \rightarrow{ }^{4} F_{3 / 2}\right)$ of $\mathrm{Nd}^{3+}: \mathrm{S}$-VAP with different concentrations.

citation source was quite high $(\sim 3 \mathrm{~W})$. When focused into the crystal this excitation could have heated the volume exposed.

As the temperature is raised to $300 \mathrm{~K}$ it appears that there are only two strong absorption lines resulting from the ${ }^{4} I_{9 / 2} \rightarrow{ }^{4} F_{3 / 2}$ transition of the main site. The other absorption lines from the secondary sites become extremely weak compared to those of the main lines, as shown in Fig. 5. The emission lines of the secondary sites are also relatively much weaker at room temperature than at $2 \mathrm{~K}$ as shown in Fig. 6 . The sharp line emissions from the secondary sites observed at $2 \mathrm{~K}$ contribute to a broader band emission observed at $300 \mathrm{~K}$.

The absorption lines $e$ and $f$ in the low temperature absorption spectrum are actually not sharp lines. They are two broad bands with strong peaks at 11607 and $11622 \mathrm{~cm}^{-1}$, and some weak structure at the higher energy side as shown in Fig. 1. At $80 \mathrm{~K}$, the whole band emits as can be seen in Fig. 4, indicated by the arrow. The excitation spectrum of the main site emission at $1.065 \mu \mathrm{m}$ was measured at $15 \mathrm{~K}$ in the vicinity of lines $e$ and $f$. The $1.065 \mu \mathrm{m}$ emission intensity along with the absorbed power is plotted as a function of excitation wavelength in Fig. 7 showing that the excitation spectrum has the same structure as the absorption spectrum. It seems that even at this low temperature they are strongly coupled and the energy transfer efficiency from line $e$ to the main site is about $100 \%$.

The excitation spectrum of the main site emission at $1.065 \mu \mathrm{m}$ was also obtained at $300 \mathrm{~K}$ in the $800 \mathrm{~nm}$ region of the ${ }^{4} I_{9 / 2} \rightarrow{ }^{4} F_{5 / 2},{ }^{2} H_{9 / 2}$ absorption and is plotted in Fig. 8 . The absorption spectra for the ${ }^{4} I_{9 / 2} \rightarrow{ }^{4} F_{5 / 2},{ }^{2} H_{9 / 2}$ transition at 2 and $300 \mathrm{~K}$ are shown in Fig. 9 for comparison. 


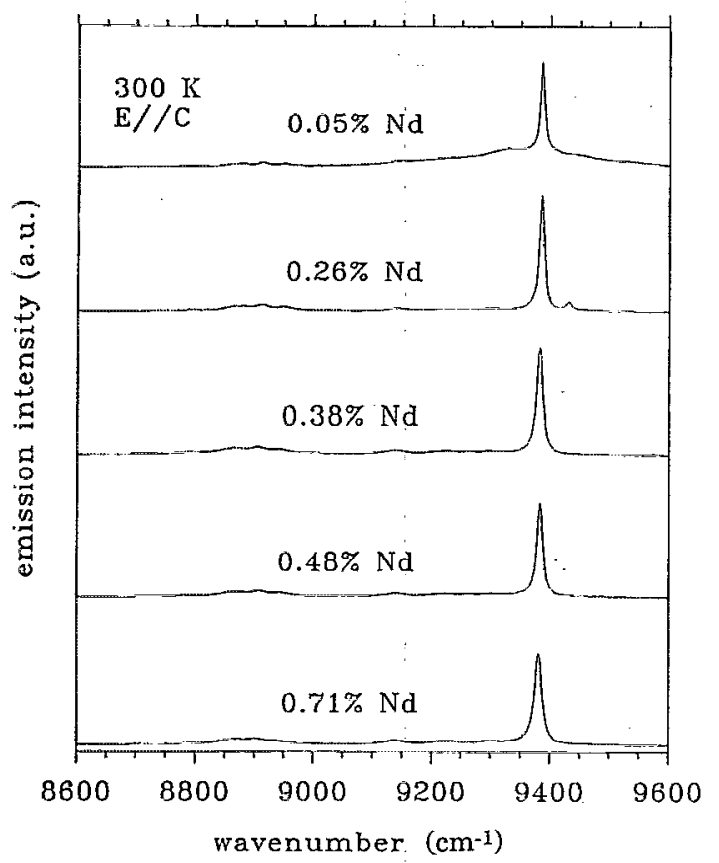

FIG. 6. Room temperature $\pi$ polarized emission spectra $\left({ }^{4} F_{3 / 2} \rightarrow{ }^{4} I_{11 / 2}\right)$ of $\mathrm{Nd}^{3+}: \mathrm{S}-\mathrm{VAP}$ with different concentrations excited by a multiline argon ion laser.

Energy levels of the main site were determined from 80 $\mathrm{K}$ absorption and emission data and are given in Table II. The absorption line $f$ is identified as the absorption to the upper ${ }^{4} F_{3 / 2}$ level which is $11624 \mathrm{~cm}^{-1}$ at this temperature. The Stark splitting of the ${ }^{4} F_{3 / 2}$ level in $\mathrm{Nd}^{3+}:$ S-VAP is $346 \mathrm{~cm}^{-1}$.

The energy levels of the secondary sites have smaller Stark splittings compared to the main site as we can see from the low temperature absorption spectra since their absorption lines lie between those of the main site. The absorption lines at the higher energy side are also broadened while the absorption lines at the lower energy side are quite sharp, which is similar to the behavior of the main site. The energy levels of the secondary sites were difficult to determine. The broad-

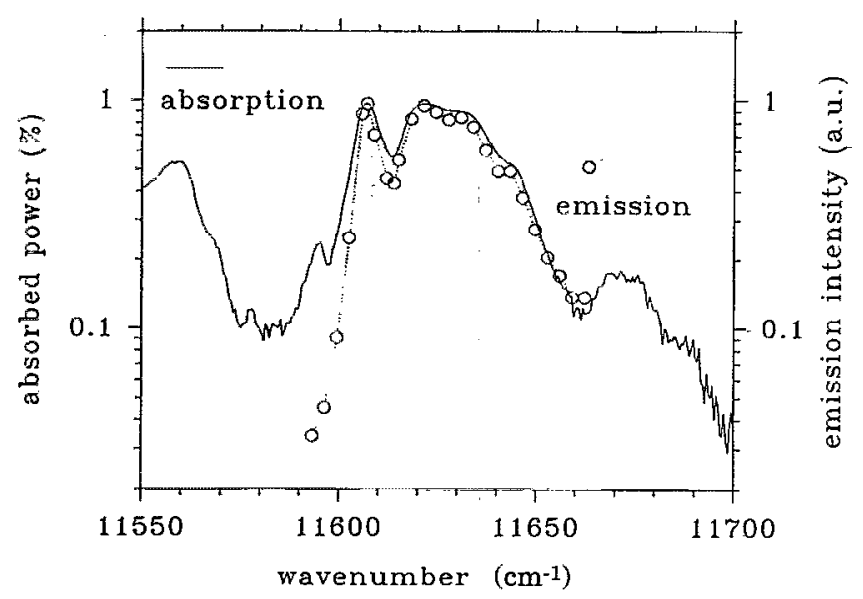

FIG. 7. Excitation spectrum of the main site emission at $1.065 \mu \mathrm{m}$ at $15 \mathrm{~K}$.

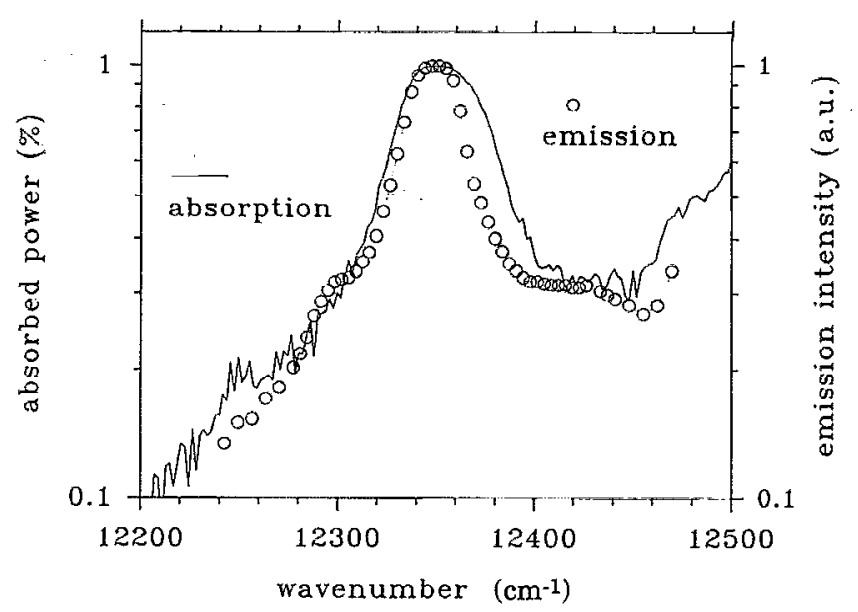

FIG. 8. Excitation spectrum of the main site emission at $1.065 \mu \mathrm{m}$ at $300 \mathrm{~K}$.

ening of the absorption at higher energy side make it very difficult to correlate the upper ${ }^{4} F_{3 / 2}$ level to the corresponding lower ${ }^{4} F_{3 / 2}$ level for each individual site. It was not possible to separate the emissions of these secondary sites in our site selective spectroscopy experiments. This may be due to the limited spectral resolution of the excitation source and/or indicate the strong coupling between the secondary sites.

The concentration dependence of the decay time of the ${ }^{4} F_{3 / 2}$ state was measurcd. The results are shown in Fig. 10 . The ${ }^{4} F_{3 / 2}$ state of $\mathrm{Nd}^{3+}: \mathrm{S}$-VAP has very strong selfabsorption. To avoid the decay time lengthening due to the self-absorption, ${ }^{8}$ luminescence decay time measurements were done with fine-ground powders of crystals. At room temperature, the luminescence decay of the ${ }^{4} F_{3 / 2}$ state is exponential for very low $\mathrm{Nd}^{3+}$ concentration with a decay time of $213 \mu \mathrm{s}$. As the $\mathrm{Nd}^{3+}$ concentration is increased the

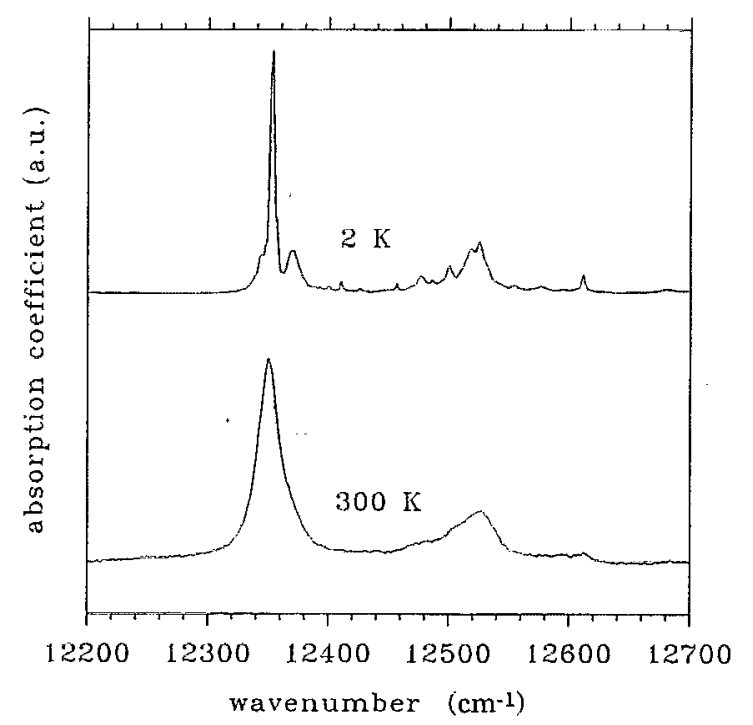

FIG. 9. $\pi$ polarized transmission spectra $\left({ }^{4} I_{9 / 2} \rightarrow{ }^{4} F_{5 / 2}\right)$ of 0.48 at. $\%$ $\mathrm{Nd}^{3+}: \mathrm{S}-\mathrm{VAP}$ at 2 and $300 \mathrm{~K}$. 
TABLE II. Energy levels of $\mathrm{Nd}^{3 *}$ ion in the main site of S-VAP at $80 \mathrm{~K}$.

\begin{tabular}{cc}
\hline Speciral term & Wave number $\left(\mathrm{cm}^{-1}\right)$ \\
\hline${ }^{4} F_{3 / 2}$ & 11624,11278 \\
${ }^{4} I_{15 / 2}$ & $6620,6506,6396,6373$, \\
& $6311,6257,6246,5716$ \\
${ }^{4} I_{13 / 2}$ & $4480,4387,4376,4353$, \\
& $4306,4261,3803$ \\
${ }^{4} I_{11 / 2}$ & $2484,2410,2391,2357$, \\
& 2319,1889 \\
${ }^{4} I_{9 / 2}$ & $667,551,499,449,0$ \\
\hline
\end{tabular}

decay becomes nonexponential and the decay time shorter. For nonexponential decay a mean decay time is defined as

$$
\tau=\frac{1}{I(0)} \int_{0}^{\infty} I(t) d t
$$

where $I(0)$ and $I(t)$ are luminescence intensities at time 0 and $t$. The decrease of the decay time with increase in $\mathrm{Nd}^{3+}$ concentration indicates the existence of concentration quenching in this crystal.

\section{LASER PERFORMANCE}

The 0.26 at. $\% \mathrm{Nd}^{3+}:$ S-VAP crystal was cut with flat and parallel faces containing the $c$ axis and antireflection (AR) coated from 1.0 to $1.1 \mu \mathrm{m}$. It was $3.8 \mathrm{~mm}$ long. The laser resonator was composed of a $5 \mathrm{~cm}$ radius of curvature reflector (high reflectivity at $1.0-1.1 \mu \mathrm{m}$ and high transmission at $790-810 \mathrm{~nm}$ ) and a flat output coupler (OC) with transmission up to $5 \%$. The pump light was focused with a $10 \mathrm{~cm}$ focal length lens through the HR mirror into the crystal. Both pulsed and cw laser-pump-laser experiments were performed in manners simulating diode laser pumping. Pulsed lasing was achieved by pumping with a long pulsed Cr:LiSAF laser which was tuned to the $\mathrm{Nd}^{3+}: \mathrm{S}-\mathrm{VAP}$ absorption peak at 810

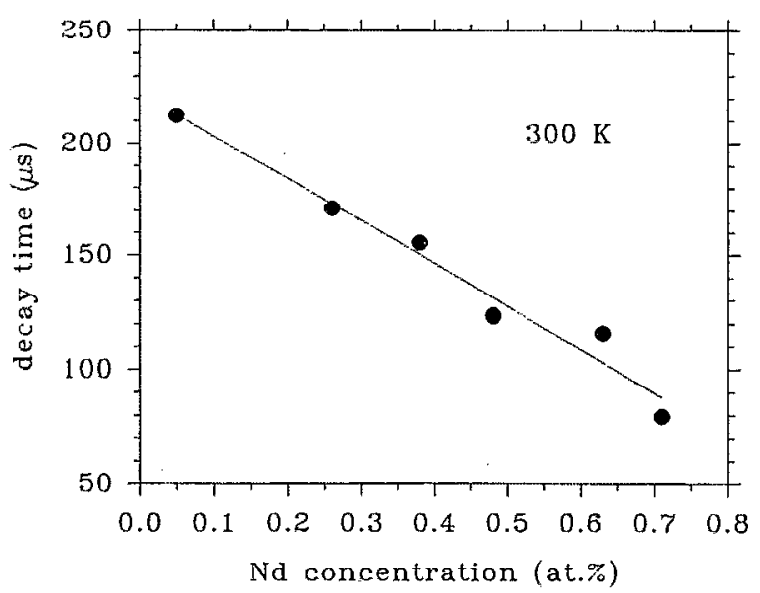

FIG. 10. Concentration dependence of the ${ }^{4} F_{3 / 2}$ state decay time of $\mathrm{Nd}^{3+}: \mathrm{S}-\mathrm{VAP}$ at room temperature.
TABLE III. Thresholds and slope efficiencies of $0.26 \% \mathrm{Nd}^{3+}$ :S-VAP lasing at $1.065 \mu \mathrm{m}$ in pulsed and $\mathrm{cw}$ operation.

\begin{tabular}{lccccc}
\hline & \multicolumn{2}{c}{ Pulsed } & & \multicolumn{2}{c}{ cw } \\
\cline { 2 - 3 } \cline { 5 - 6 }$T(\%)$ & $\begin{array}{c}\text { Threshold } \\
(\mu \mathrm{J})\end{array}$ & $\begin{array}{c}\text { Slope efficiency } \\
(\%)\end{array}$ & $\begin{array}{c}\text { Threshold } \\
(\mathrm{mW})\end{array}$ & $\begin{array}{c}\text { Slope efficiency } \\
(\%)\end{array}$ \\
\hline 1.2 & 3.6 & 38 & & 5.8 & 29 \\
3 & 5.2 & 59 & & 9.4 & 43 \\
5 & 6.0 & 62 & 13.2 & 49 \\
\hline
\end{tabular}

$\mathrm{nm}$ and had a spectral bandwidth of about $1 \mathrm{~nm}$. cw lasing was achieved using a $\mathrm{cw}$ Ti:sapphire laser tuned to $810 \mathrm{~nm}$ with a spectral bandwidth of about $0.1 \mathrm{~nm}$. Lasing from the $\mathrm{Nd}^{3+}: \mathrm{S}-\mathrm{VAP}^{4} F_{3 / 2} \rightarrow{ }^{4} I_{11 / 2}$ transition at $1.065 \mu \mathrm{m}$ is linearly polarized along the $c$ axis ( $\pi$ polarization).

About $85 \%$ of the long pulsed pumping energy and $98 \%$ of cw pumping power were absorbed in the laser crystal. The thresholds and slope efficiencies for both pulsed and $\mathrm{cw}$ operation at $1.065 \mu \mathrm{m}$ are listed in Table III as a function of output coupling transmission. With a $5 \%$ output coupler, a slope efficiency of $62 \%$ and $49 \%$ was obtained in pulsed and cw operation, respectively. Higher slope efficiency is expected with a higher transmission output coupler. ${ }^{3,4}$

A $1.3 \mathrm{~mm} 0.65$ at. $\% \mathrm{Nd}^{3+}$-doped S-VAP crystal was also tested for laser performance in the same cavity. A slope efficiency of only $22 \%$ was obtained with a $5 \%$ output coupler in cw operation. The thresholds and slope efficiencies of this crystal in pulsed and cw operation are listed in Table IV as a function of output coupler transmission.

\section{DISCUSSION}

The main site, $M_{\mathrm{II}}$, for the $\mathrm{Nd}^{3+}$ ion in S-VAP is the site of the lasing ions. Room-temperature emissions originate principally from ions in the $M_{\text {II }}$ site while the other lines seen at $20 \mathrm{~K}$ contribute to a broad emission band (see Fig. 6). The peak absorption cross section of the ${ }^{4} I_{9 / 2} \rightarrow{ }^{4} F_{5 / 2},{ }^{2} H_{9 / 2}$ transition at $809.6 \mathrm{~nm}$ is $3 \times 10^{-19} \mathrm{~cm}^{2}$ with a linewidth around $2 \mathrm{~nm}$. The emission from the ${ }^{4} F_{3 / 2} \rightarrow{ }^{4} I_{11 / 2}$ transmission of the main site is highly polarized in $\pi$ polarization $(E / / c)$ and has an emission cross section ${ }^{3}$ of $5 \times 10^{-19} \mathrm{~cm}^{-2}$. The emission is dominated by one single narrow emission line at $1.065 \mu \mathrm{m}$ having a line width of $1.2 \mathrm{~nm}$.

As we can see in Fig. 9 the absorption spectra which would enable diode laser pumped lasing at $300 \mathrm{~K}$ actually contains absorptions of the main site and the secondary sites, i.e., the wide absorption width at room temperature is partly due to absorption by $\mathrm{Nd}^{3+}$ ions in the secondary sites. It is

TABLE IV. Thresholds and slope efficiencies of $0.65 \% \mathrm{Nd}^{3+}$ :S-VAP lasing at $1.065 \mu \mathrm{m}$ in pulsed and $\mathrm{cw}$ operation.

\begin{tabular}{cccccc}
\hline & \multicolumn{2}{c}{ Pulsed } & & \multicolumn{2}{c}{$\mathrm{cw}$} \\
\cline { 2 - 3 } \cline { 5 - 6 }$T(\%)$ & $\begin{array}{c}\text { Threshold } \\
(\mu \mathrm{J})\end{array}$ & $\begin{array}{c}\text { Slope efficiency } \\
(\%)\end{array}$ & $\begin{array}{c}\text { Threshold } \\
(\mathrm{mW})\end{array}$ & $\begin{array}{c}\text { Slope efficiency } \\
(\%)\end{array}$ \\
\hline 1.2 & 5.0 & 23 & 9.5 & 11 \\
3 & 10.0 & 36 & & 16.4 & 18 \\
5 & 13.0 & 46 & 19.8 & 22 \\
\hline \hline
\end{tabular}




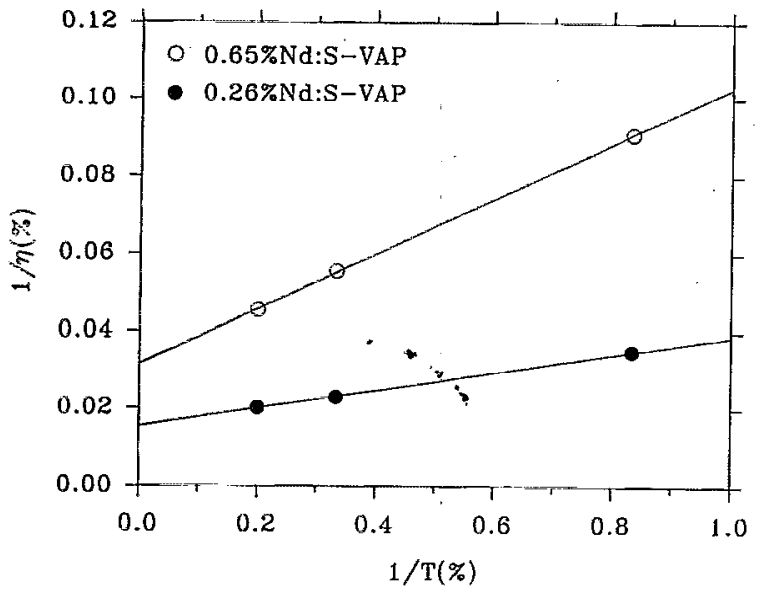

FIG. 11. Inverse slope efficiency versus inverse output coupling for $0.26 \%$ $\mathrm{Nd}^{3+}$ and $0.65 \% \mathrm{Nd}^{3+}$-doped S-VAP laser crystals when lasing at $1.065 \mu \mathrm{m}$.

clear that the absorption of the secondary sites, which is on the sides of the absorption band, does not contribute to the $1.065 \mu \mathrm{m}$ emission as efficiently as the main site absorption, as demonstrated in the excitation spectrum of Fig. 8. In the other words, the absorption of the secondary sites reduces the pump efficiency. Therefore, this crystal is not appropriate for broadband pumping. The wide absorption width of $\mathrm{Nd}^{3+}$-doped S-VAP (about $2 \mathrm{~nm}$ ) is not an advantage for this crystal since it results from contributions from inequivalent sites. It is an extreme case of inhomogeneous broadening.

In a $\mathrm{cw}$ laser-pumped laser experiment the slope efficiency obtained depends, under the condition of $100 \%$ pump efficiency, on the quantum efficiency, the pump and lasing wavelengths, and the loss present in the laser material in the following relation: ${ }^{2}$

$$
\eta=\left(\eta_{q} \lambda_{p} / \lambda_{l}\right) T /(T+L),
$$

where $\eta_{q}$ is the quantum efficiency $\lambda_{p}$ and $\lambda_{l}$ are the pump and lasing wavelengths, $T$ is the output coupling, and $L$ is the cavity loss. $1 / \eta$ is linearly dependent on $1 / T$, which can be seen if Eq. (2) is inverted to give,

$$
1 / \eta=1 / \eta_{0}+\left(L / \eta_{0}\right) 1 / T,
$$

where

$$
\eta_{0}=\eta_{q} \lambda_{p} / \lambda_{l}
$$

is the intrinsic slope efficiency (i.e., is the slope efficiency when there is no loss present). For $\mathrm{Nd}^{3+}$-doped S-VAP the pumping and lasing wavelengths are 810 and $1065 \mathrm{~nm}$, respectively. Therefore, we have $\lambda_{p} / \lambda_{l}=76 \%$, and the intrinsic slope efficiency is proportional to the quantum efficiency. ${ }^{2}$

The loss present in laser performance can be derived from the slope efficiency obtained in the $\mathrm{cw}$ laser-pumped laser experiment using Eq. (3). The plot of the inverse output coupling versus the inverse slope efficiency for 0.26 at. \% $\mathrm{Nd}^{3+}$-doped S-VAP, Fig. 11, gives an intrinsic slope efficiency of $67 \%$ and a passive loss of $2.0 \% \mathrm{~cm}^{-1}$ for cw operation. The plot of the inverse output coupling against the versus slope efficiency for 0.65 at. $\% \mathrm{Nd}^{3+}$-doped S-VAP crystal is also given in Fig. 11. An intrinsic slope efficiency of $32 \%$ and a passive loss of $8.9 \% \mathrm{~cm}^{-1}$ is obtained.

In the presence of concentration quenching the quantum efficiency is equal to the ratio of the mean decay time to the radiative decay time. For 0.26 at. $\% \mathrm{Nd}^{3+}$-doped S-VAP, the mean decay time of the $1.065 \mu \mathrm{m}$ emission is $171 \mu \mathrm{s}$. Then the quantum efficiency is equal to $80 \%$. But for 0.65 at. \% $\mathrm{Nd}^{3+}$-doped S-VAP the mean decay time is only $128 \mu \mathrm{s}$, which gives a quantum efficiency of $60 \%$. Lower quantum efficiency will result in lower intrinsic slope efficiency and, consequently, lower measured slope efficiency. ${ }^{2}$ Therefore, the slope efficiency of the 0.65 at. $\% \mathrm{Nd}^{3+}$-doped S-VAP is expected to be lower than that of the 0.26 at. $\% \mathrm{Nd}^{3+}$-doped $\mathrm{S}$-VAP. That partially cxplains the lower slope efficiency we obtained form the 0.65 at. \% crystal. Also the crystal quality of the high concentration sample is not as good as the low concentration one, which results in higher passive loss and contributes to the decreased slope efficiency.

\section{SUMMARY}

In summary, we have investigated the spectroscopic properties and laser performance of the newly discovered laser crystal, $\mathrm{Nd}^{3+}$-doped $\mathrm{Sr}_{5}\left(\mathrm{VO}_{4}\right)_{3} \mathrm{~F}$. Detailed spectroscopic studies show that there exist at least 11 spectroscopically different $\mathrm{Nd}^{3+}$ sites although there are only two inequivalent crystallographic sites $\left(M_{\mathrm{I}}\right.$ and $M_{\mathrm{II}}$ sites) where $\mathrm{Nd}^{3+}$ ions can reside in this crystal. The strongest absorption and emission observed result from the transition of $\mathrm{Nd}^{3+}$ ions in $M_{\text {II }}$ sites, the most common or main site. $\mathrm{Nd}^{3+}$ ions in this site are responsible for the lasing action. The multisite nature is believed to result from the doping of $\mathrm{Nd}^{3+}$ ions in $M_{\mathrm{I}}$ sites and the inequivalent charge compensation induced by this doping. Although the site selective spectroscopy indicates that there is energy transfer from the secondary sites to the main site, the excitation spectrum taken in the vicinity of diode laser pumping wavelength shows that this transfer efficiency is quite low. The pump efficiency, and thus the lasing efficiency, will be reduced by the absorption of the secondary sites in the vicinity of the diode laser wavelength. Therefore, excellent laser performance is not expected for broadband pumping although high slope efficiency and low threshold laser performance of both pulsed and cw operation in low $\mathrm{Nd}^{3+}$ concentration $\mathrm{Sr}_{5}\left(\mathrm{VO}_{4}\right)_{3} \mathrm{~F}$ crystal at $1.065 \mu \mathrm{m}$ have been achieved when pumped with narrow band pulsed $\mathrm{Cr}$ :LiSAF and cw Ti:sapphire lasers. Concentration quenching was observed in high $\mathrm{Nd}^{3+}$ concentration crystals, and it is shown to degrade the laser performance in this crystal.

\section{ACKNOWLEDGMENTS}

This work was supported by the Advanced Research Projects Agency (ARPA) and by the Florida High Technology and Industry Council. The fabrication of crystals and the mirror coatings supplied by Lightning Optical Corp. is gratefully appreciated.

${ }^{1}$ X. X. Zhang, P. Hong, G. Loutts, J. Lefaucheur, M. Bass, and B. H. T. Chai, Appl. Phys. Lett. 64, 3205 (1994).

${ }^{2}$ X. X. Zhang, G. B. Loutts, M. Bass, and B. H. T. Chai, Appl. Phys. Lett. 64, 10 (1994). 
${ }^{3}$ S. A. Payne, B. H. T. Chai, W. L. Kway, L. D. DeLoach, L. K. Smith, G. Lutts, R. Peale, X. X. Zhang, G. D. Wilke, and W. F. Krupke, Conference on Lasers and Electro-Optics (CLEO), Baltimore, 1993, postdeadline paper CPD12.

${ }^{4}$ B. H. T. Chai, G. Lutts, R. Peale, X. X. Zhang, S. A. Payne, W. F. Krupke, L. D. DeLoach, and L. K. Smith, Opt. Lett. (to be published).
${ }^{5}$ D. A. Grisafe and F. A. Hummel, J. Solid State Chem. 2, 160 (1970).

${ }^{6}$ R. C. Ohlmann, K. B. Steinbruegge, and R. Mazelsky, Appl. Opt. 7, 905 (1968).

${ }^{7}$ G. B. Loutts and B. H. T. Chai, Proc. SPIE 1863, 31 (1993).

${ }^{8} \mathrm{X}$. X. Zhang, P. Hong, B. Di Bartolo, C. W. Struck, and M. Bass, J. Phys.:Condens. Matter 6, 4661 (1994). 